\title{
Oxygenation of carbon nanotubes: Atomic structure, energetics, and electronic structure
}

\author{
S. Dag, ${ }^{1}$ O. Gülseren, ${ }^{1,2,3}$ T. Yildirim, ${ }^{2}$ and S. Ciraci ${ }^{1}$ \\ ${ }^{1}$ Department of Physics, Bilkent University, Ankara 06800, Turkey \\ ${ }^{2}$ NIST Center for Neutron Research, National Institute of Standards and Technology, Gaithersburg, Maryland 20899 \\ ${ }^{3}$ Department of Materials Science and Engineering, University of Pennsylvania, Philadelphia, Pennsylvania 19104 \\ (Received 12 April 2002; revised manuscript received 24 July 2002; published 30 April 2003)
}

\begin{abstract}
This paper presents an extensive and systematic analysis of the oxygenation of semiconducting and metallic single-wall carbon nanotubes by using the first principles pseudopotential plane wave method. Our study involves the physisorption of oxygen molecules, chemisorption of oxygen atoms and formation of an oxide, and deals with the equilibrium binding geometry and corresponding electronic energy structure. The binding energies of an oxygen molecule physisorbed at different sites are determined by calculating short and long range interactions. The triplet state of the physisorbed oxygen molecule is energetically favorable, whereas the nonmagnetic (spin paired) state yields a relatively stronger binding energy. An oxygen atom is adsorbed on top of the carbon-carbon bond. The zigzag bonds of the nanotubes are weakened and eventually are replaced by a carbon-oxygen-carbon bridge bond. Chemisorption of atomic oxygen and physisorption of an oxygen molecule modify the electronic energy structure of the bare tube in different ways. For a different coverage and pattern, self-consistent field electronic energy structure calculations using the optimized physisorption geometry corresponding to the triplet ground state result in a small energy gap between unoccupied oxygen levels and the top of the valence band of the semiconducting carbon nanotube. These results invalidate the hole doping of the semiconducting carbon nanotube upon the physisorption of oxygen.
\end{abstract}

DOI: 10.1103/PhysRevB.67.165424

PACS number(s): 73.22.-f, 61.48.+c, 73.20.Hb, 71.30.+h

\section{INTRODUCTION}

Single-wall carbon nanotubes ${ }^{1}$ (SWNTs) are quasi-one dimensional nanowires and exhibit either metallic or semiconducting properties depending on their chirality and radius. ${ }^{2-5}$ One of the grand challenges of research on carbon nanotubes has been the realization of nanometer optoelectronic devices. ${ }^{6-8}$ Proposed device operations on a SWNT have utilized the contacts to metal substrates and the local modification of electronic properties by introducing a chemical doping profile or local defects, elastic radial or other types of deformation, and also by the adsorption of foreign atoms (functionalization) ${ }^{9-14}$ In particular, the search for new properties to be used in technological applications has made the functionalization of carbon nanotubes a subject of both experimental and theoretical interest. Recently, the modification of electronic properties of carbon nanotubes by the adsorption of foreign atoms or molecules has been actively studied. ${ }^{14-17}$ Hydrogen chemisorption can give rise to dramatic effects on the electronic and atomic structure. ${ }^{14}$ It has been predicted that, depending on the hydrogen decoration, the tube can undergo changes between a wide band gap insulator and a metal with a high density of states at the Fermi level. ${ }^{18} \mathrm{~A}$ well-defined pattern of hydrogen adsorption can change the circular cross section to a square one ${ }^{18}$ Remarkable effects on the electrical resistance of a semiconducting single wall carbon nanotube (s-SWNT) upon exposure to gaseous molecules such as $\mathrm{NO}_{2}$ and $\mathrm{NH}_{3}$, have been reported. ${ }^{15}$ Collins et al. ${ }^{16}$ found similar effects for oxygen. Exposure to air or oxygen dramatically influences electrical resistance and the thermoelectric power of a single wall carbon nanotubes. A s-SWNT, which can be converted to a good metal, and whose electronic properties can be reversibly modified by surprisingly small concentration of adsorbed oxygen has been proposed as a candidate for chemical sensor devices. Also the metallic connects to a device made from a SWNT could easily be realized by physisorption of $\mathrm{O}_{2}$. Experimental studies on carbon nanotube field emitters have shown that the adsorption of ambient gases, in particular $\mathrm{O}_{2}$ instantaneously induces a significant increase in the emission current. ${ }^{19}$ In addition to functionalization, oxygenation involves other applications. For example, carbon nanotubes synthesized by using arc discharge are purified from other undesired, carbon based nanoparticles through oxidation. At elevated temperatures, oxygen undergoes a chemical reaction preferably with the strained $\mathrm{C}-\mathrm{C}$ bonds and eliminates carbonaceous nanoparticles as well as the caps of nanotubes. ${ }^{20-22}$

Observed effects on the electronic structure of SWNTs due to $\mathrm{O}_{2}$ physisorption have been subject to recent theoretical investigations based on first-principles calculations. ${ }^{23-30}$ Spin-unpolarized band structure calculations based on the local density approximation (LDA) predicted that the semiconducting $(8,0)$ tube becomes metallic, since the valence band is hole doped by the Fermi level touching the top of the valence band as a result of $\mathrm{O}_{2}$ physisorption. ${ }^{23}$ The analysis based on the local spin density approximation (LSDA) has indicated that the physisorbed $\mathrm{O}_{2}$ favors the triplet state. While the spin-up states are fully occupied, the spin-down states are nearly empty and hence give rise to finite density of states at the Fermi level. ${ }^{23}$ The binding geometry and the corresponding electronic energy structure of the $\mathrm{O}_{2}$ + SWNT system, based on the first principles and fully relaxed, spin polarized calculations including the long range interactions, have not been thoroughly investigated yet. Therefore, the previous, spin unpolarized calculations of the electronic structure may not show the real effect of $\mathrm{O}_{2}$ physisorption on the electronic structure of a s-SWNT. The en- 
ergetics of oxygen adsorption on the surface of graphite ${ }^{24}$ and on a $(8,0)$ SWNT were calculated for selected sites. ${ }^{25}$ Adsorption and desorption of an oxygen molecule and various precursor states at the edges of finite size armchair $(5,5)$ and zigzag $(9,0)$ SWNTs were studied to provide an understanding of oxidative etching process. ${ }^{26}$ Similarly, the mechanism of the oxidative etching of the caps and walls of the small radius $(5,5)$ armchair SWNT was investigated. ${ }^{27}$ The effects of oxygen adsorption on the field emission from carbon nanotubes were treated by using an $a b$ initio approach. ${ }^{28}$ The dynamics of thermal collision of $\mathrm{O}$ atom with a $(6,0)$ SWNT is simulated by $a b$ initio calculations. ${ }^{29}$

In this paper we present a detailed, first-principles analysis of the oxygen adsorption on the SWNTs. Our prime motivation is to reveal how the adsorption of $\mathrm{O}_{2}$ and $\mathrm{O}$ modifies the electronic properties. The zigzag $(8,0)$ and armchair $(6,6)$ tubes are taken as prototype tubes for semiconducting (s-SWNT) and metallic (m-SWNT) single-wall carbon nanotubes, respectively. We expect similar trends in other tubes with curvature effects being emphasized at small radii. ${ }^{5}$ In Sec. II, we summarize the methods used in the calculations. In Sec. III we investigate the character of bonding and energetics in the adsorption of $\mathrm{O}_{2}$. We calculate binding energy for $\mathrm{O}_{2}$ adsorbed at different sites and coverage for both magnetic (spin-polarized) and nonmagnetic (spin-unpolarized) states within the density functional theory (DFT) ${ }^{31}$ We find that the $\mathrm{O}_{2}+\mathrm{SWNT}$ system in the triplet state becomes energetically more favorable, but the weak binding of $\mathrm{O}_{2}$ on a SWNT is further weakened in the magnetic state. Moreover, in the magnetic state a small band gap opens between the top of the valence band of s-SWNT and the spin-up states of $\mathrm{O}_{2}$. Previous first-principles calculations dealing with the physisorption of $\mathrm{O}_{2}$ have omitted the van der Waals ( $\mathrm{vdW}$ ) interaction. Here, in addition to the short range (chemical) interaction, we calculated the long range vdW interaction for different adsorption sites of the $\mathrm{O}_{2}$. In Sec. IV, we study the chemisorption of atomic $\mathrm{O}$ at different sites and found that at certain conditions a strained zigzag $\mathrm{C}-\mathrm{C}$ bond is replaced by a strong $\mathrm{C}-\mathrm{O}-\mathrm{C}$ bridge bond upon the chemisorption of $\mathrm{O}$ atom. Results of electronic structure calculations for $\mathrm{O}_{2}$ + SWNT systems with different patterns and coverages are given in Sec. V. Discussion of results and conclusions are presented in Sec. VI.

\section{METHOD}

The first principles total energy and electronic structure calculations have been performed using the pseudopotential plane wave method ${ }^{32}$ within the generalized gradient approximation (GGA). ${ }^{33}$ We carried out both spin unpolarized and spin polarized calculations using a periodically repeating tetragonal supercell with lattice constants $a_{s c}, b_{s c}$, and $c_{s c}$. The lattice constants, $a_{s c}$ and $b_{s c}$, are chosen such that the interaction between nearest neighbor tubes is negligible (the minimum $\mathrm{C}-\mathrm{C}$ distance between two nearest neighbor tubes is taken as $\sim 10 \AA$ ). The lattice constant along the axis of the tube, $c_{s c}$, is taken to be equal to the one-dimensional lattice parameter $c$ of the tube (which is specified as single supercell). To minimize the adsorbate-adsorbate interaction, some calculations are performed in longer supercells by taking $c_{s c}=2 c$ (double supercell). We used ultra soft pseudopotentials for carbon and oxygen atoms ${ }^{34}$ and plane waves up to an energy cutoff of $400 \mathrm{eV}$. Owing to the very large lattice constants of the supercell, $a_{s c}$ and $b_{s c}$, k-point sampling is done only along the tube axis. The Monkhorst-Pack special k-point scheme $e^{35}$ is used with 12 and $6 \mathbf{k}$ points for single and double supercells, respectively. For all systems we studied all atomic positions of adsorbate and SWNTs, as well as $c$ are fully optimized by using the conjugate gradient method.

Previous DFT calculations have shown that the GGA generally yields a better cohesive energy than LDA which usually overbinds. However, relative to the GGA, the LDA predicts a smaller lattice constant (or bond distance) and a larger bulk modulus. ${ }^{36,37}$ In particular, for graphite we found that the LDA cohesive energy was larger than both the GGA and experiment, but interlayer distance calculated by the GGA was much larger than experiment, as well as the LDA. The ratio of lattice parameters of graphite, $c_{g} / a_{g}$, have been found to be 2.73 and 3.73 for the LDA and GGA, respectively. The corresponding experimental value ${ }^{38}$ at zero temperature is 2.72. Upon including the long range interactions described below, while the GGA value improves to 2.73 , the LDA value is lowered to 2.47 . This clearly shows that the LDA overbinds. These trends are in agreement with those found by Janotti et al. ${ }^{39}$ and Furthmüller et al. ${ }^{40}$. It is well known that both the GGA and LDA take into account only short range (chemical) interactions, but exclude weak, long range van der Waals interaction. However neither of these approximation describe correctly the asymptotic bonding behavior of neutral systems. The contribution of weak $\mathrm{vdW}$ interaction to the binding energy is usually omitted in the chemisorption of atoms or molecules resulting in strong bonding. The situation is, however, different in the case of the physisorption where the contribution of short range and long range interactions are comparable. Therefore, in treating the binding energy of the physisorbed $\mathrm{O}_{2}$ we include the attractive $\mathrm{vdW}$ interaction, and calculate the corresponding energy, $E_{v d W}=\Sigma_{i j} C_{6 i j} / r_{i j}^{6}$, using the asymptotic form of the Lifshitz's formula. ${ }^{41-43}$ We assign a positive sign to $E_{v d W}$ since stable binding is specified by positive energies in the present study. Here $r_{i j}$ is the distance between the $i$ th $\mathrm{O}$ atom and the $j$ th $\mathrm{C}$ atom, and the constant $C_{6 i j}$ is calculated within the Slater-Kirkwood approximation ${ }^{44}$ to be $10.604 \mathrm{eV}(\AA) .{ }^{6}$ We note, however, that this standard calculation of the $\mathrm{vdW}$ energy may involve ambiguities due to the relatively small distance between the physisorbed molecule and the SWNT. Nevertheless, the vdW interaction is attractive and strengthens the chemical bond.

\section{PHYSISORPTION OF $\mathrm{O}_{2}$ MOLECULES}

We studied the bonding of $\mathrm{O}_{2}$ by placing the molecule at different sites on the SWNT, and by calculating the binding energy corresponding to the optimized structure. Different physisorption positions described in Fig. 1, i.e., on top of the axial C-C bond ( $A$ site), above the center of the hexagonal carbon rings ( $H$ site), on top of the zigzag $\mathrm{C}-\mathrm{C}$ bond $(Z$ site), 
$\mathbf{A}$

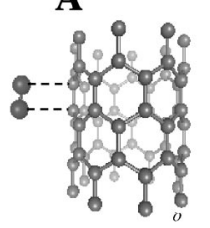

$d_{c-o}=2.90 \stackrel{\circ}{A}$

$E_{s}=-5 \mathrm{meV}$

$E$, $=120 \mathrm{meV}$
$\mathbf{H}$

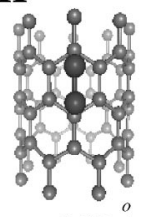

$d_{c-o}=2.89 \AA$

$E_{s}=4 \mathrm{meV}$

$E_{\mathrm{h}}=159 \mathrm{meV}$

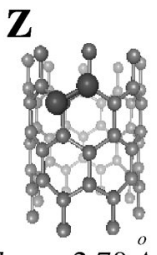

$d_{c-o}=2.70 \AA$

$E_{s}=-27 \mathrm{meV}$

$E_{\mathrm{h}}=158 \mathrm{meV}$

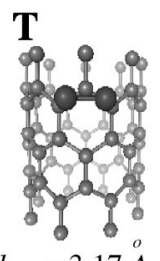

$d_{c-o}=3.17 \stackrel{\circ}{A}$

$E_{s}=37 \mathrm{meV}$

$E_{h}=191 \mathrm{meV}$
FIG. 1. Schematic description of the physisorption sites of $\mathrm{O}_{2}$ molecule on the $(8,0)$ SWNT. Geometrical data and binding energies corresponding to these sites are given in Table I. The GGA optimized distance from one $\mathrm{O}$ atom of the molecule to the nearest $\mathrm{C}$ atom of the SWNT is denoted by $d_{C-O} . E_{s}$ is the GGA chemical bonding energy for the spin polarized triplet state. $E_{b}$ is the binding energy including the van der Waals interaction.

and perpendicular to the axis of the tube and above two adjacent zigzag bonds ( $T$ site) were considered.

The binding energy involves short and long range interactions, i.e., $E_{b}=E_{s}+E_{v d W}$. The contribution of the short range interaction (i.e., chemical bonding energy) is calculated by using the expression

$$
E_{s}=E_{T}[\mathrm{SWNT}]+E_{T}\left[O_{2}\right]-E_{T}\left[O_{2}+\mathrm{SWNT}\right]
$$

in terms of the GGA total energies of the fully optimized bare SWNT $\left(E_{T}[\mathrm{SWNT}]\right)$, the molecule $\left(E_{T}\left[O_{2}\right]\right)$, and $\mathrm{O}_{2}$ physisorbed on the SWNT $\left(E_{T}\left[O_{2}+\mathrm{SWNT}\right]\right)$, which are calculated by using the same (single or double) supercell and calculational parameters. By definition, $E_{s}>0$ in Eq. (1) corresponds to a stable and exothermic chemical bonding. In some cases, $E_{s}<0$ is endothermic, but it corresponds to a local minimum where the desorption of $\mathrm{O}_{2}$ from the SWNT is prevented by a barrier. Note that the calculation of the bare molecules in the same supercell excludes the contribution of the nearest neighbor $\mathrm{O}_{2}-\mathrm{O}_{2}$ coupling in $E_{s}$. Our results for the bond dissociation energies of $\mathrm{O}_{2}$ obtained from the double supercell are 8.60 and $5.86 \mathrm{eV}$ for spin unpolarized and spin polarized triplet state calculations, respectively. The triplet state is the ground state of $\mathrm{O}_{2}$. The experimental bond energy of $\mathrm{O}_{2}$ in the triplet state, $5.2 \mathrm{eV}^{45}$ is in fair agreement with the calculated value of $5.86 \mathrm{eV}$ found in this study.

The vdW energies are calculated for a single $\mathrm{O}_{2}$ physisorbed on a $(8,0)$ SWNT with a cutoff distance of 1000 unit cells. Using the binding geometries determined from the minimization of GGA total energies, $E_{T}\left[O_{2}+\mathrm{SWNT}\right]$, we calculated $E_{v d W}$ 's to be $125,155,185$, and $155 \mathrm{meV}$ for $A$, $H, Z$, and $T$ sites, respectively. When we repeat the same calculations for a s-SWNT of 100 unit cell long, these wdW energies changed only by $10^{-4} \mathrm{meV}$. We believe that this is a reasonable convergence for a tube having finite length. By adding these $E_{v d W}$ values to those corresponding GGA chemical bonding energies, $E_{s}$ we obtain binding energies $E_{b}$ of physisorbed $\mathrm{O}_{2}$ for different sites. Calculated binding energies of the physisorbed $\mathrm{O}_{2}$ by using single and double supercells at various sites for spin unpolarized and spin polarized states are listed in Table I. Our calculations of the
TABLE I. Calculated binding energies $E_{b}$ of an $\mathrm{O}_{2}$ molecule at different sites of the $(8,0)$ SWNT. Different sites of physisorption, $A, H, Z$, and $T$ sites, are described in Fig. 1. Calculation of GGA chemical bonding energies, $E_{s}$ are performed by using single (i.e., $c_{s c}=c$ ) and double (i.e., $c_{s c}=2 c$ ) supercells. In the third column $u$, $s$, and $t$ indicate spin-unpolarized, spin-polarized singlet state and triplet calculations, respectively. The GGA optimized distance from one $\mathrm{O}$ atom of the molecule to the nearest $\mathrm{C}$ atom of SWNT is denoted by $d_{C-O}$. The average bond length of $\mathrm{O}_{2}$ is $1.24 \AA$. The GGA chemical bonding energies $E_{s}$ exclude the $\mathrm{O}_{2}-\mathrm{O}_{2}$ coupling energy except one given in the parentheses. The binding energies, $E_{b}$ in the sixth column includes the van der Waals interactions.

\begin{tabular}{lccccc}
\hline \hline site & supercell & state & $d_{C-O}(\AA)$ & $E_{s}(\mathrm{meV})$ & $E_{b}(\mathrm{meV})$ \\
\hline$A$ & single & $u$ & 2.87 & $122(221)$ & \\
& single & $t$ & 2.87 & -32 & \\
& single & $s$ & 2.87 & -0.5 & \\
& double & $u$ & 2.90 & 141 & \\
& double & $t$ & 2.90 & -5 & 120 \\
$H$ & double & $u$ & 2.89 & 155 & \\
& double & $t$ & 2.89 & 4 & 159 \\
$Z$ & double & $u$ & 2.70 & 187 & \\
& double & $t$ & 2.70 & -27 & 158 \\
$T$ & double & $u$ & 3.17 & 172 & \\
& double & $t$ & 3.17 & 37 & 191 \\
\hline \hline
\end{tabular}

binding energy as a function of the distance between $\mathrm{O}_{2}$ and SWNT, $E_{b}(d)=E_{s}(d)+E_{v d W}(d)$, indicates that under the $\mathrm{vdW}$ interaction the equilibrium distance moves towards the surface of the SWNT. Since $E_{s}(d)$ decreases much faster than $E_{v d W}(d)$ increases with decreasing $d$, the equilibrium distance occurs $\sim 0.2 \AA$ less than the GGA optimized distance. Note that in these calculations GGA and vdW interactions are treated in different levels of approximation; while the first one is calculated from the first-principles, the latter is empirical and is derived from the Lifshitz's asymptotic formula which is valid for large $d$. Similar arguments are also valid for the equilibrium distance calculated within LDA $+\mathrm{vdW}$. The latter approximation is expected to yield relatively smaller equilibrium distance than the GGA+vdW.

The small binding energies in Table I are characteristics of physisorption. Although the spin unpolarized state of the $\mathrm{O}_{2}+$ SWNT system is energetically unfavorable, we first discuss it for comparison with previous calculations. The strongest binding for spin unpolarized calculations $(u)$ occurs at $\mathrm{Z}$-site with $E_{s}=187 \mathrm{meV}$, where $\mathrm{O}_{2}$ is placed on top of (and parallel to) a zigzag $\mathrm{C}$-C bond. The stability of the $Z$-site physisorption is tested by starting from a configuration, where the $\mathrm{O}_{2}$ molecule is rotated from the equilibrium position by $90^{\circ}$, and hence becomes perpendicular to the underlying zigzag bond. Upon relaxing, the molecule rotated towards its original equilibrium, Z-site, position. The GGA chemical bonding energy of the $A$-site physisorption is calculated to be $122 \mathrm{meV}$ from a single supercell. However, this energy increases to $221 \mathrm{meV}$ when the $\mathrm{O}_{2}-\mathrm{O}_{2}$ coupling energy in the single cell is not subtracted from $E_{s}$. This can be achieved by taking $E_{T}\left[\mathrm{O}_{2}\right]$ in Eq. (1) calculated from the double cell as the energy of the isolated $\mathrm{O}_{2}$ molecule energy. 
TABLE II. Calculated C-O distance $d_{C-O}$, chemical bonding energy $E_{s}$, van der Waals energy $E_{v d W}$, and binding energy $E_{b}$ for $\mathrm{O}_{2}$ physisorbed on the $H$, and $B$ sites, of $(6,6)$ armchair m-SWNT.

\begin{tabular}{lcccc}
\hline \hline site & $d_{C-O}(\AA)$ & $E_{s}(\mathrm{meV})$ & $E_{v d W}(\mathrm{meV})$ & $E_{b}(\mathrm{meV})$ \\
\hline $\mathrm{H}$ & 3.04 & -19 & 151 & 132 \\
$\mathrm{~B}$ & 2.92 & -32 & 138 & 106 \\
\hline \hline
\end{tabular}

The GGA spin unpolarized chemical bonding energy of $\mathrm{O}_{2}$ at A-site is calculated in the double cell and is found to be 141 $\mathrm{meV}$, i.e., $\sim 20 \mathrm{meV}$ larger than that of the single supercell value. This suggests that the bonding between $\mathrm{O}_{2}$ and the SWNT becomes relatively stronger in the absence of $\mathrm{O}_{2}-\mathrm{O}_{2}$ coupling, since the charge rearrangement is affected in the presence of the latter interaction. Using a similar firstprinciples method within the local density approximation Jhi et $a l .{ }^{23}$ found $E_{s}=250 \mathrm{meV}$ for the A-site physisorption. We repeated our calculations by using the LDA, and found $E_{s}$ $=204 \mathrm{meV}$. Our LDA results are in fair agreement with that of Jhi et $a .^{23}$ and also confirm that LDA yields overbinding as compared to GGA. Surprisingly, Moon et al. ${ }^{27}$ did not report a physisorbed state of $\mathrm{O}_{2}$ on the $(5,5)$ armchair SWNT. For higher coverage, we considered that all $A$ sites in a unit cell of a s-SWNT are filled by physisorbed $\mathrm{O}_{2}$ molecules. The energy $E_{s}$ relative to the spin-unpolarized energy of an individual $\mathrm{O}_{2}$ molecule is found to be larger than 400 $\mathrm{meV}$ per molecule from the single supercell. In compliance with the above arguments, the increased value of $E_{s}$ with respect to the single $A$-site physisorption is due to the increased $\mathrm{O}_{2}-\mathrm{O}_{2}$ coupling, which is not subtracted from the chemical bonding energy.

Spin polarized calculations yield relatively lower (stronger) total energies, $E_{T}\left[\mathrm{O}_{2}+\mathrm{SWNT}\right]$, and hence set the triplet state as the ground state with a net magnetic moment of $\sim 2 \mu_{B}$ per unitcell. In this case, GGA chemical bonding energies are generally weakened and at $A$ and $Z$ sites; they become even negative with $E_{s}^{t}=-5$ and $-27 \mathrm{meV}$, respectively. The spin polarized calculations yield that the $T$ site is the energetically most favorable site with $E_{s}^{t}=37 \mathrm{meV}$. Then the binding energy $E_{b}$ is found to be $191 \mathrm{meV}$ by adding $E_{v d W}=154 \mathrm{meV}$ to $E_{s}$. This binding energy is in agreement with the recent measuremnt by Ulbricht et al. ${ }^{46}$ We note that these binding energies are small, and becomes exothermic mainly owing to the long range vdW interaction.

We also studied the physisorption of $\mathrm{O}_{2}$ on the $(6,6)$ armchair SWNT to reveal the effect of metallicity of the tube on physisorption of $\mathrm{O}_{2}$. We consider two possible physisorption sites; i.e., above the center of the hexagon ( $H$ site), and on top of the $\mathrm{C}-\mathrm{C}$ bond and perpendicular to the axis of the tube ( $B$ site). The $B$ site of a $(6,6)$ tube is similar to the $A$ site of a $(8,0)$ tube, but in the former case the $\mathrm{C}-\mathrm{C}$ bonds under the adsorbed $\mathrm{O}_{2}$ is highly strained, since it lies on the circumference. The binding structure and binding energies calculated with a spin polarized GGA are given in Table II.

\section{CHEMISORPTION OF OXYGEN ATOMS}

Atomic oxygen is reactive and highly electronegative. The ground state of the atomic oxygen is the triplet state, and its energy is found $0.93 \mathrm{eV}$ lower than the singlet state. It forms strong bonds with the substrate atoms and eventually oxidizes the surface. For example, atomic $\mathrm{O}$ may break the $\mathrm{Si}-\mathrm{Si}$ bond, and form a $\mathrm{Si}-\mathrm{O}-\mathrm{Si}$ on the surface of $\mathrm{Si}$. In this respect, the effect of the adsorbed oxygen on the structural and electronic properties of a SWNT is important. The breaking of the O-O bond of a physisorbed $\mathrm{O}_{2}$ molecule is unlikely owing to the weak interaction with the SWNT. However, it was shown that near the defect sites of the graphite surface $\mathrm{O}_{2}$ molecule can dissociate. ${ }^{24}$ A carbon nanotube, that can be visualized as a graphene rolled into a cylinder, is normally more reactive than the surface of graphite. As a result, $\mathrm{O}_{2}$ physisorbed near the defect sites of a SWNT is expected to dissociate into atomic oxygens. ${ }^{47}$ In fact, it was shown that there is no activation barrier for dissociation of $\mathrm{O}_{2}$ when it is adsorbed at the zigzag edge of a SWNT. ${ }^{26}$ Owing to the concerted motion of the atoms at the proximity of the molecule and energy gained by the individual oxygen atoms engaging in the bonding with the SWNT concomitant with the dissociation, the activation energy for dissociation is expected to be low. In this section, we study the interaction between atomic O and SWNT, and reveal the nature of the chemical bonding. We consider only the short range interaction, and hence the resulting chemisorption energies $E_{s}$ for the following reasons. First, the chemisorption energies are rather high (in the range of $5 \mathrm{eV}$ ), and hence much larger than the vdW energies. Second, Lifshitz's asymptotic formula may not be appropriate for relatively smaller inter atomic distances $r_{i j}$, such as $d_{C-O}$ $\sim 1.5 \AA$, and yields energies which may give rise to misleading conclusions. ${ }^{43}$ For example, we calculated $E_{v d W}$ of $\mathrm{O}$ atom chemisorbed at the a-site to be $2.39 \mathrm{eV}$.

Various adsorption sites and summary of our results are shown in Fig. 2. Spin-polarized calculations yield the singlet state with a net zero magnetic moment as the ground state. Since free oxygen atom has different energies for its different states (i.e. the $E_{T}^{t}[O]$ triplet state, the $E_{T}^{s}[O]$ singlet state, and the $E_{T}^{u}[O]$ spin unpolarized state), $E_{s}$ of chemisorbed $\mathrm{O}$ calculated from Eq. (1) depends on which reference state is taken. For example, for the a-site chemisorption one can give $E_{s}^{t t}=1.88 \mathrm{eV}, \quad E_{s}^{s t}=3.16 \mathrm{eV}, \quad E_{s}^{s s}=4.09 \mathrm{eV}, \quad$ and $E_{s}^{u}$ $=5.04 \mathrm{eV}$. Chemisorption energies calculated with respect to different reference states are listed in Table III. In Fig. 2, the chemisorption energies $E_{s}$ obtained from the spin unpolarized calculations are shown. Interestingly, spinunpolarized calculations are resulted with approximately the same total energy as the singlet state. On the other hand, the total energy of the triplet state for the a site is found to be $1.28 \mathrm{eV}$ higher (energetically less favorable) than that of the singlet state.

Among all sites considered in this study the single $\mathrm{O}$ adsorbed on top of the zigzag C-C bond (i.e. $\mathbf{z}$ site) is energetically most favorable with $E_{s}=E_{s}^{u}=5.07 \mathrm{eV}$. The hollow (c) site, i.e. the center of the hexagons on the surface of the SWNT, appears to be a local minimum with relatively smaller $E_{s}$. Whereas the adsorption on top of carbon atoms does not correspond to a local minimum, the $\mathrm{O}$ atom moves toward the center of neighboring C-C bonds. Apparently, 
a

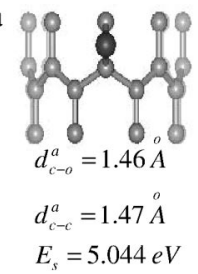

aa

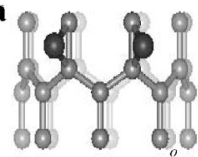

$d^{a}=1.46 \stackrel{o}{A}$

$d_{c-c}^{a}=1.41 \stackrel{o}{A}$

$E_{\mathrm{s}}=4.923 \mathrm{eV}$

az2

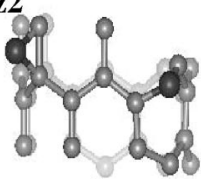

$d_{c-o}^{a}=1.45 \stackrel{\circ}{A}, d_{c-o}^{z}=1.38 \stackrel{\circ}{\AA}$

$d_{c-c}^{a}=1.47 \stackrel{\circ}{A}, d_{c-c}^{z}=2.15 \AA$

$E_{\mathrm{s}}=5.256 \mathrm{eV}$

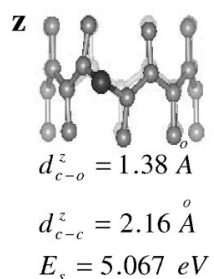

c
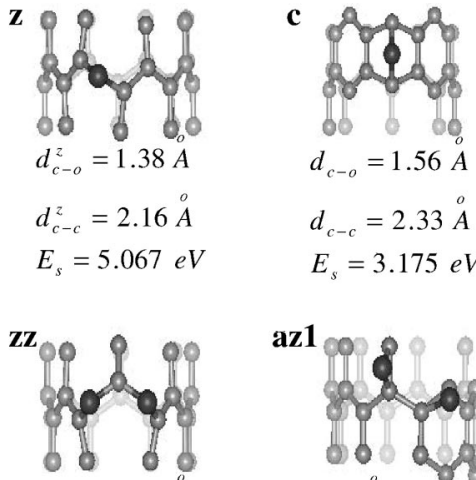

$d_{c-o}^{z}=1.37 \AA$

$d_{c-c}^{z}=2.24 \stackrel{A}{A}$

$E_{s}=5.284 \mathrm{eV}$

aa1

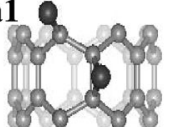

$d_{i-o}^{z}=1.45 \stackrel{\circ}{A}$

$d_{c-c}^{z}=1.46 \stackrel{\circ}{A}$

$E_{s}=4.983 \mathrm{eV}$

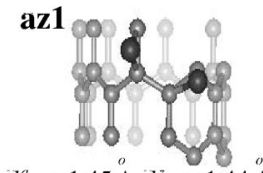

$d_{c-o}^{a}=1.45 \AA{ }^{\circ}, d_{c-o}^{z}=1.44 \stackrel{\circ}{A}$

$d_{c-c}^{a}=1.46 \stackrel{\circ}{A}, d_{c-c}^{z}=1.53 \AA$

$E_{s}=4.939 \mathrm{eV}$
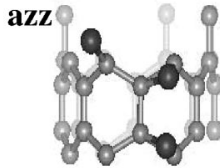

$d_{c-o}^{a}=1.45 \stackrel{\circ}{A}, d_{c-o}^{z}=1.40^{\circ} \AA$

$d_{c-c}^{a}=1.46 \stackrel{\circ}{A}, d_{c-c}^{z}=1.51 \stackrel{\circ}{\AA}$

$E_{s}=4.635 \mathrm{eV}$

FIG. 2. Schematic description of the various adsorption sites of atomic $\mathrm{O}$ on the $(8,0) \mathrm{SWNT}$. Some relevant geometrical data and GGA chemical bond (chemisorption) energies, corresponding to these sites are also given. $E_{s}$ : chemisorption energy; $d_{C-O}$ : length of the $\mathrm{C}-\mathrm{O}$ bond; $d_{C-C}$ : length of the $\mathrm{C}-\mathrm{C}$ bond under adsorbed $\mathrm{O}$ atom. $E_{s}$ is obtained from the spin-unpolarized calculations of the total energies in Eq. (1).

atomic oxygen favors the bonding on top of a $\mathrm{C}-\mathrm{C}$ bond, where strong $\mathrm{C}-\mathrm{O}$ bonds can form. The binding energies, as large as $\sim 5 \mathrm{eV}$, suggest that atomic $\mathrm{O}$ is, actually chemisorbed with a significant charge transfer from $\mathrm{C}$ to $\mathrm{O}$. Moreover, the energy gained from the chemisorption of two atomic oxygen is more than the bond energy of $\mathrm{O}_{2}$ in either magnetic or non-magnetic state. This implies the dissociation of $\mathrm{O}_{2}$ followed by the chemisorption of individual $\mathrm{O}$ atoms is an exothermic process similar to other oxidation processes.

The binding energies of $\mathrm{O}$ at $\mathbf{a}$, and $\mathbf{z}$ sites are comparable, but the length of the $\mathrm{C}-\mathrm{C}$ bond under adsorbed $\mathrm{O}$ is

TABLE III. Calculated chemical bonding energies of chemisorbed $\mathrm{O}$ atom at $\mathbf{a}$ and $\mathbf{z}$ sites. For $E_{s}^{s t}$ and $E_{s}^{s s}$ the ground state for the oxygen chemisorbed SWNT is the singlet state, but the isolated $\mathrm{O}$ atom (reference state) is in the triplet state and the singlet state, respectively. $E_{s}^{t t}$ corresponds to the both isolated $\mathrm{O}$ and $\mathrm{O}$ chemisorbed SWNTs in the triplet state. $E_{s}^{u}$ stands for the spinunpolarized calculation. The energy unit is $\mathrm{eV}$.

\begin{tabular}{lcc}
\hline \hline & a site & z site \\
\hline$E_{s}^{s t}$ & 3.16 & 3.18 \\
$E_{s}^{s s}$ & 4.09 & 4.11 \\
$E_{s}^{u}$ & 5.04 & 5.07 \\
$E_{s}^{t t}$ & 1.88 & \\
\hline \hline
\end{tabular}

different for these different sites. The zigzag bond is elongated to $2.16 \AA$ upon $\mathrm{O}$ chemisorption at the $\mathbf{z}$ site, while the length of the axial $\mathrm{C}-\mathrm{C}$ bond is practically unaltered after $\mathrm{O}$ chemisorption at the a site. The elongation of the zigzag bond followed by the contraction of the $\mathrm{C}-\mathrm{O}$ bonds indicates that the $\mathrm{C}-\mathrm{C}$ bond of the bare SWNT is either broken or weakened after the chemisorption of $\mathrm{O}$. One can argue that at the a site the $\mathrm{C}-\mathrm{C}$ bond could have not broken, if it is constrained by the periodic boundary condition. Binding energies $\left(E_{s}=4.72,4.99,5.03\right.$, and $\left.4.84 \mathrm{eV}\right)$ and stress $(\sigma$ $=-45.9,-19.2,9.2$, and $37.8 \mathrm{kB})$ calculated for different values of lattice parameter $(c=4.35,4.30,4.25$, and $4.20 \AA$ ) invalidate this argument, and confirm the fact that $c$ changes slightly upon the chemisorption of a single $\mathrm{O}$ atom. Then the question as to why the C-C bond at the $\mathbf{z}$ site is broken upon the chemisorption of $\mathrm{O}$, but that at the a site, can be answered by the fact that the zigzag bonds are highly strained and hence their $s p^{2}$ character are modified. ${ }^{17,5}$ This is an important manifestation of the curvature effect. ${ }^{4}$ Experimentally, it was shown that oxygen exposure first oxidizes, and eventually etches away the nanotube with the smaller radius. ${ }^{48}$ Earlier, we showed that the binding energy of $\mathrm{Al}$ and $\mathrm{H}$ increases at the high curvature site of a SWNT under a circumferential elliptic deformation. ${ }^{17}$ It is, therefore, expected that the reactivity of the SWNT for oxygen chemisorption, even the dissociation of an $\mathrm{O}_{2}$ molecule, can be enhanced at the high curvature sites which is realized by the applied radial deformation. The charge density contour plots on planes passing through the $\mathrm{O}$ atom chemisorbed at either a or $\mathbf{z}$ sites and the underlying $\mathrm{C}-\mathrm{C}$ bond are compared in Fig. 3. In the case of a-site chemisorption we see that the $\mathrm{C}-\mathrm{C}$ bond survives with a characteristic bonding charge, but new $\mathrm{C}-\mathrm{O}$ bonds are formed. On the other hand, while the $\mathrm{C}-\mathrm{C}$ bond of $\mathbf{z}$-site is weakened, even is broken, the $\mathrm{C}-\mathrm{O}$ bonds become stronger as compared to the similar bonds at a-site chemisorption.

Other relevant sites and configurations in $\mathrm{O}$ chemisorption are also included in Fig. 2. The binding energies do not change significantly in the case of more $\mathrm{O}$ atoms are chemisorbed at neighboring sites. Notably, the total binding energy of two $\mathrm{O}$ atoms chemisorbed on top of adjacent zigzag bonds (i.e., zz-site chemisorption) is $\sim 10.4 \mathrm{eV}, 4.5 \mathrm{eV}$ higher than the bond dissociation energy of the bare $\mathrm{O}_{2}$ molecule in the triplet state. Therefore the chemisorption of two $\mathrm{O}$ atoms following the dissociation of physisorbed $\mathrm{O}_{2}$ molecule in the triplet state is exothermic. The comparison of the az1- and az2-site chemisorption reveals that the zigzag $\mathrm{C}-\mathrm{C}$ bond is stabilized by an adjacent a-site chemisorption. However, this bond is broken when $\mathbf{a}$ - and $\mathbf{z}$-site chemisorptions are farther apart. In the azz configuration the average binding energy of three $\mathrm{O}$ atoms is lowered, but is still close to the binding energy of a single $\mathrm{O}$. This implies that higher coverage of $\mathrm{O}$ atom on the SWNT can be stable. We examined two different high oxygen coverage corresponding to the oxidation of the nanotube. These are half coverage, (where oxygen atoms are adsorbed to all the a sites) and full coverage, (where oxygens are adsorbed to all $\mathbf{a}$ and $\mathbf{z}$ sites of SWNT). The half coverage was stable with an average binding energy of $4.96 \mathrm{eV}$. In the case of full coverage, $\mathrm{O}_{2}$ molecules reformed due to the 

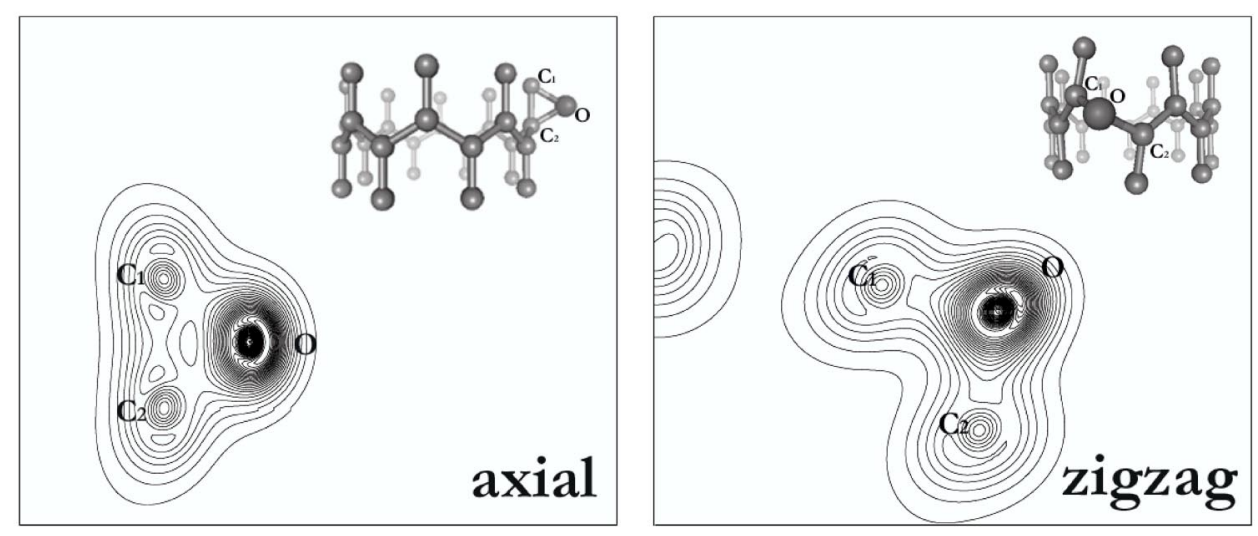

FIG. 3. Charge density contour plots on a plane containing an $\mathrm{O}$ atom and the nearest $\mathrm{C}-\mathrm{C}$ bond in the case of a-site chemisorption (a) and $\mathbf{z}$-site chemisorption (b). These chemisorption sites and their atomic configuration are described as insets. increased interaction between closely lying adsorbed $\mathrm{O}$ atoms.

\section{ELECTRONIC STRUCTURE}

In the previous sections we studied the atomic structure and energetics of $\mathrm{O}_{2}$ and $\mathrm{O}$ adsorption on a s-SWNT and a $\mathrm{m}-\mathrm{SWNT}$, and revealed various stable adsorption sites (and patterns) at different coverage. In this section, we will examine the electronic structure corresponding to these adsorption patterns. Because of supercell method used in the present study we obtain energy bands and density of states corresponding to a periodically repeating adsorption pattern. For the energy level structure of a single adsorbate, the adsorbate-adsorbate interaction indigenous to the supercell method can be reduced by taking relatively longer cell sizes, allowing longer nearest neighbor distances. Under these circumstances the bands are flatten and represent the energy level of the dopant.

First we studied the electronic energy structure of an oxygen molecule physisorbed on A-site of $(8,0)$ SWNT. Although A-site is not energetically most favorable site we consider it in order to compare our results with those of Jhi et al., ${ }^{23}$ who performed first-principles LDA and LSDA calculations for a single $\mathrm{O}_{2}$ physisorbed per unit cell.

In Fig. 4(a) we present the band structure of the bare $(8,0)$ SWNT, which is a semiconductor with a band gap $E_{g}$ $\sim 0.7 \mathrm{eV}$ at $\Gamma$-point between the bottom of the conduction band $E_{C}$ and the top of the valence band $E_{V}$. The $\pi^{*}-\sigma^{*}$ hybridized singlet state is in the conduction band. ${ }^{4,5}$ The free linear chain of $\mathrm{O}_{2}$ molecules, in principle, has a weak bonding state with an equilibrium lattice parameter slightly larger than $c$. In Fig. 4(b), the free linear chain of $\mathrm{O}_{2}$ in registry with the $(8,0)$ tube has half filled doubly degenerate $p p \pi^{*}$ bands. For the triplet state, (which is energetically more favorable than the spin unpolarized state as well as singlet state) these bands split into two doubly degenerate bands $\left[p p \pi^{*}(\uparrow)\right.$ and $\left.p p \pi^{*}(\downarrow)\right]$. The $p p \pi^{*}(\uparrow)$ bands are filled and separated from the empty $p p \pi^{*}(\downarrow)$ bands by an energy gap of $\sim 2 \mathrm{eV}$. In Fig. 4(c) spin unpolarized GGA calculations with $c_{s c}=c$ yield doubly degenerate, half filled $p p \pi^{*}$ bands in the band gap for the physisorption of $\mathrm{O}_{2}$ at the $A$ site. The Fermi level touching the top of the valence band $E_{V}$ makes the system metallic.
The above situation is, however, changed in the spin polarized calculations, which yields the triplet state as the ground state. Under these circumstances two $\mathrm{O} p p \pi^{*}$ bands split into four bands; two of them occur $\sim 2 \mathrm{eV}$ below the valence band of s-SWNT. The remaining two empty bands rise $350 \mathrm{meV}$ above $E_{V}$ at the $\Gamma$ point and make a band gap of $90 \mathrm{meV}$ [see Fig. 4(d)]. Therefore, the hole doping picture developed from the LDA and LSDA calculations ${ }^{23}$ does not appear in the present spin polarized GGA calculation, since the bands of the triplet ground state open a band gap. To reveal the source of disagreement between the present GGA

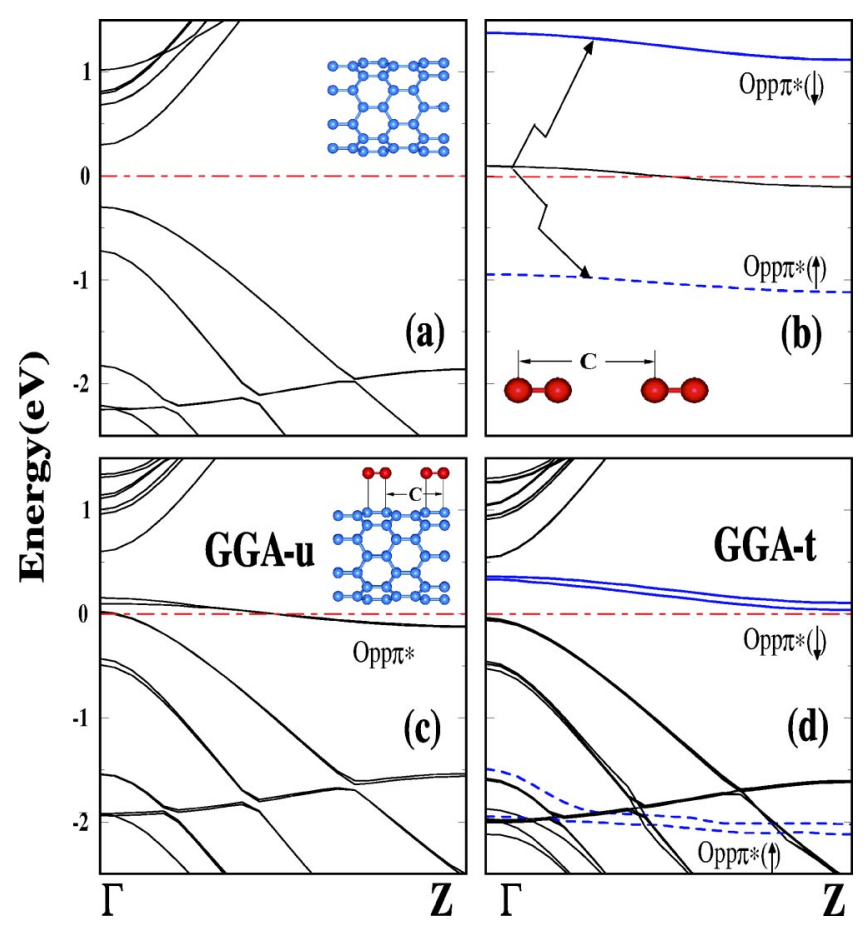

FIG. 4. (a) Spin unpolarized energy bands of the $(8,0)$ bare s-SWNT; (b) spin polarized (dashed lines) and spin unpolarized (solid lines) energy bands of the linear $\mathrm{O}_{2}$ chain with the same lattice parameter $c$; (c) spin unpolarized energy bands of the $\mathrm{O}_{2}$ physisorbed on the $(8,0)$ tube with $O_{p p \pi^{*}}$ state pinning the Fermi level. (d) Spin polarized bands corresponding to (c). Zero of energy is taken at the Fermi level shown by the dash-dotted line. Up-spin and down-spin bands are indicated by corresponding arrows. Here $c_{s c}=c$. 
results and the LSDA results of Jhi et al. ${ }^{23}$ we carried out LDA and LSDA calculations (with the binding geometry used by Jhi $e t$ al. $^{23}$ and also with fully relaxed atomic structure, and using different types of pseudopotentials and cutoff energies). We found that all our LDA (spin-unpolarized) calculations are in agreement with our spin-unpolarized GGA calculations yielding $\mathrm{O} p p \pi^{*}$ band overlapping with the top of the s-SWNT valence band, and hence are confirming the results of Jhi et al. ${ }^{23}$ However, all our LSDA calculations (corresponding to the triplet ground state) have resulted in a significant band gap (of 140-90 meV) agreeing with the present spin polarized GGA results.

Note that the bands here are only the artifact of the supercell method, and hence in the absence of band dispersion the energy level due to the single physisorbed $\mathrm{O}_{2}$ and the top of the s-SWNT valence band, $E_{V}$ shall be relatively larger than the calculated band gap. Our arguments are better explained by double cell calculations in which the $\mathrm{O}_{2}-\mathrm{O}_{2}$ coupling is reduced due to the large distance $(\sim 7 \AA)$ between nearest molecules. In Fig. 5 we summarize our results for spin unpolarized and triplet ground state bands obtained from double cell calculations. Owing to relatively large $\mathrm{O}_{2}-\mathrm{O}_{2}$ distance, the bands related with the physisorbed molecule can be taken as if the energy levels of the single adsorbate. We see that spin-paired bands in Figs. 5(a)-5(d) (which are in fact energetically unfavorable) comply with the hole doping picture $^{23}$ for all sites except the $Z$ site. For the latter, half filled $\mathrm{O}_{2}$ states are $0.2 \mathrm{eV}$ above $E_{V}$. On the other hand, the situation is rather different for triplet ground state bands illustrated in Figs. 5(e)-5(h). A sizable energy band gap of $0.2-0.4 \mathrm{eV}$ occurs between $E_{V}$ and the unoccupied spindown bands $\left[O p p \pi^{*}(\downarrow)\right.$ states] of oxygen molecule. This situation eliminates the hole doping picture. The dispersionless $O p p \pi^{*}(\downarrow)$ bands indicate that the coupling between $\mathrm{O}_{2}$ molecules are negligible.

The effects of band formation are examined by studying the electronic energy structure corresponding to various patterns of physisorbed $\mathrm{O}_{2}$. In Fig. 6 we show the band structures and density of states calculated for the zigzag chain and row of $\mathrm{O}_{2}$ physisorbed on an $(8,0) \mathrm{s}-\mathrm{SWNT}$ in the triplet ground state. In the zigzag chain, $\mathrm{O}_{2}$ molecules are placed initially above the adjacent axial C-C bonds (i.e. A sites), but upon relaxation they are tilted by $40^{\circ}$ and inclined side ways to increase O-O separation of nearest molecules. We calculated the chemical bonding energy $E_{s}=56 \mathrm{meV} / \mathrm{molecule}$. Since the number of $\mathrm{O}_{2}$ molecules per single cell is doubled, the $O p p \pi^{*}(\uparrow)$ and $O p p \pi^{*}(\downarrow)$ bands are doubled as compared to those illustrated in Fig. 4(d). The system is a semiconductor with a direct band gap $E_{g} \sim 0.4 \mathrm{eV}$. As clearly seen from the total and partial density of states the $O p p \pi^{*}$ bands are split by $\sim 2.5 \mathrm{eV}$ upon spin polarization. The band structure of the $\mathrm{O}_{2}$ row in Fig. 6(b) displays a slightly different situation. Here the gap between the lowest $O p p \pi^{*}(\downarrow)$ band and $E_{V}$ reduced to $25 \mathrm{meV}$ at the $Z$ point; it is so far the smallest band gap we obtained from spin polarized calculations. While this band complies with the hole doping picture since its is in the range of thermal excitation at room temperature, the metallization of the s-SWNT is still too far.

The electronic energy structure of higher $\mathrm{O}_{2}$ coverage was

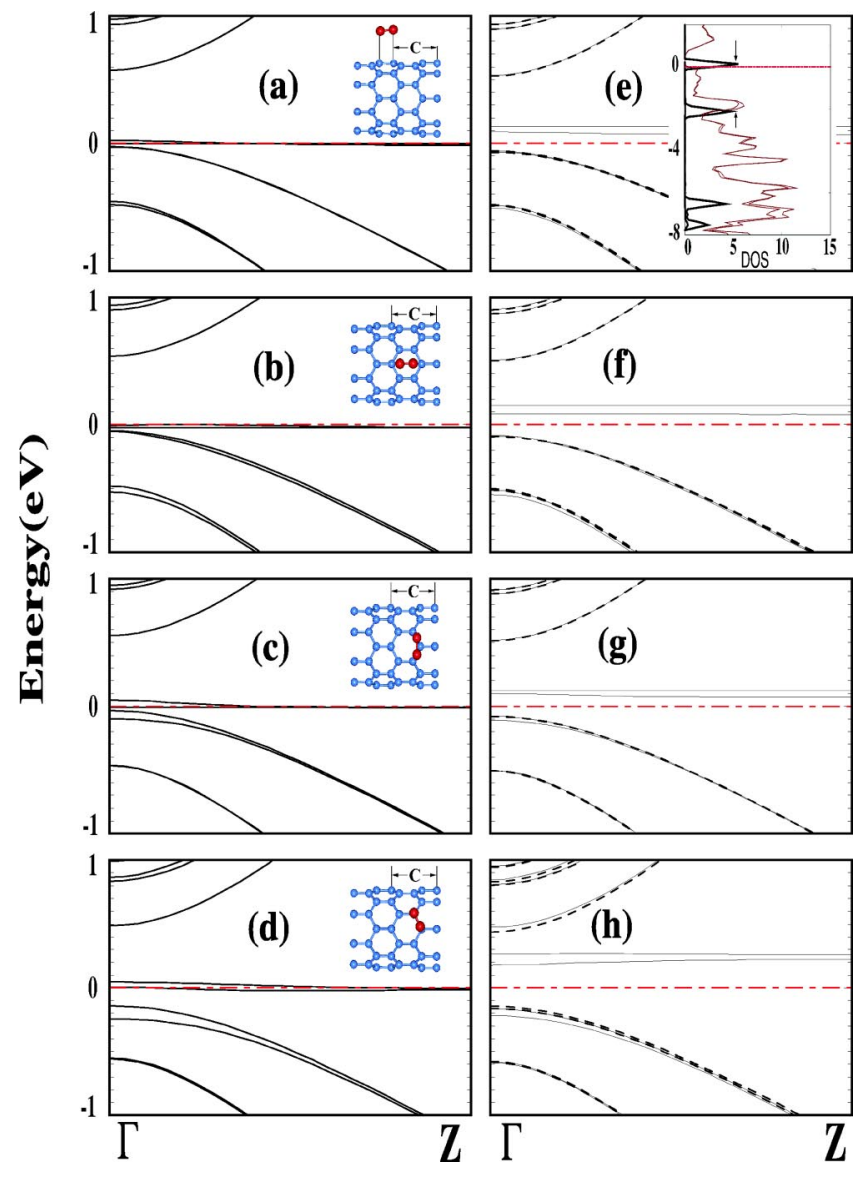

FIG. 5. Spin unpolarized and spin polarized bands of $\mathrm{O}_{2}$ physisorbed on different sites of $(8,0)$. calculations are performed by using double cells. The adsorption sites are shown by insets. (a) Spin-unpolarized and (e) triplet state bands for $A$-site physisorption. The total density of states with thin and dashed lines, and partial density of states of adsorbed $\mathrm{O}_{2}$ with thick lines are presented in panel (e). The zero of energy is taken at the Fermi level indicated by dash-dotted lines. (b) spin-unpolarized and (f) triplet state bands for the $H$ site. (c) and (g) are same for the $T$ site; (d) and (h) for the $Z$ site. For the triplet state in left panels, the spin-up and spin-down bands are shown by dashed and thin lines.

further analyzed by a system where initially $\mathrm{O}_{2}$ molecules are physisorbed to all $A$ sites of the $(8,0)$ tube. Upon relaxation of the system, the distance between $\mathrm{O}_{2}$ molecule and SWNT surface has increased continuously. This indicates that under increased $\mathrm{O}_{2}-\mathrm{O}_{2}$ coupling the chemical bonding is weakened and eventually molecules start to escape from the surface of a s-SWNT. The vdW interactions was needed to keep the molecules attached to the s-SWNT. At each step of ionic relaxation, the bands derived from the bare s-SWNT and molecular oxygen are clearly identified. This situation suggests a negligible mixing between $\mathrm{O}_{2}$ and s-SWNT states. In fact, the bands of the bare s-SWNT remain practically unchanged, and the states of $\mathrm{O}_{2}$ are broadened into bands owing to the intermolecular coupling. Several bands derived from $O p p \pi^{*}(\uparrow)$ states form a sharp peak at $\sim 2 \mathrm{eV}$ below $E_{V}$. Empty bands derived from $O p p \pi^{*}(\downarrow)$ states always occur always above $E_{V}$, and appear as another peak. Even in full coverage we see that empty $\mathrm{O}_{2}$ states and highest filled 


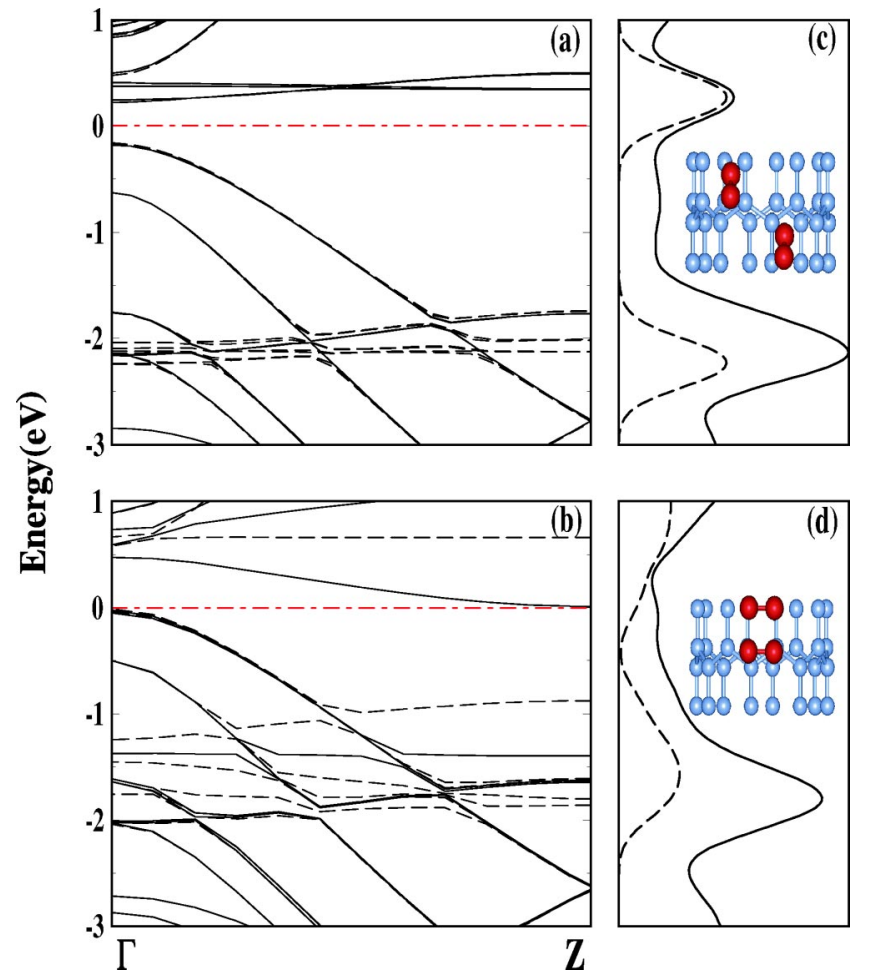

FIG. 6. (a) Spin polarized electronic energy band structure of the zigzag chain of $\mathrm{O}_{2}$ adsorbed above the adjacent axial $\mathrm{C}-\mathrm{C}$ bonds along the axis of a $(8,0)$ s-SWNT as shown by the inset. Solid and dashed lines are the spin-down and spin-up bands. The zero of energy is taken at the Fermi level $E_{F}$ indicated by the dash-dotted line. (b) Corresponding total density of states of s-SWNT $+\mathrm{O}_{2}$ and partial density of states on the oxygen atoms are shown by solid and dashed lines. (c) Band structure of the row of $\mathrm{O}_{2}$ physisorbed at $T$ sites as shown by the inset. (d) Same as (b).

s-SWNT states are separated by a gap of $\sim 100 \mathrm{meV}$, so that the electron transfer from a SWNT to empty $\mathrm{O}_{2}$ states and hence the metallization of the tube is prohibited. Under these circumstances, the s-SWNT $+\mathrm{O}_{2}$ system can be viewed by two semiconductors forming a two-liquid system. The first one is due to an $\mathrm{O}_{2}$; the second one is due to a s-SWNT. The excitation of the electrons from s-SWNT bands to $\mathrm{O}_{2}$ states may mediate intermixing of these two liquids.

The energy band structure of a m-SWNT exhibits interesting modifications upon $\mathrm{O}_{2}$ physisorption. In Fig. 7 we show the band structure of $\mathrm{O}_{2}$ adsorbed at the $H$ site of the metallic $(6,6)$ armchair tube. Similar to previous cases, here the $\mathrm{O}_{2}+(6,6)$ system is in the triplet ground state. The flat and unoccupied bands derived from the Opp $\pi^{*}(\downarrow)$-states occur $0.25 \mathrm{eV}$ above the Fermi level. The degeneracy of $\pi$-, $\pi^{*}(\downarrow)$ bands of the bare $(6,6)$ tube at the Fermi level, is lifted and a band gap of $\sim 0.4 \mathrm{eV}$ opens upon the physisorption of $\mathrm{O}_{2}$. However, the $\mathrm{O}_{2}+(6,6)$ system is still metal, since half filled $\pi^{*}(\uparrow)$ and $\pi(\uparrow)$ bands of $(6,6)$ tube cross at the Fermi level. The form and character of energy bands for $\mathrm{O}_{2}$ physisorbed at the $B$ site are essentially similar to those of the bands illustrated in Fig. 7.

Electronic states of oxygen atom chemisorbed on a SWNT depends on the coverage and pattern of adsorption.

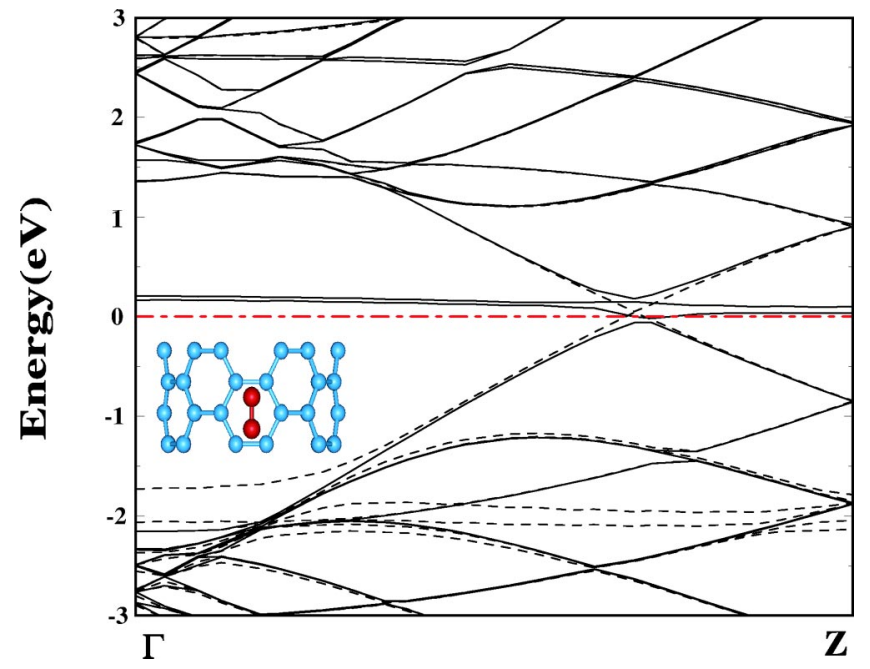

FIG. 7. Electronic energy band structure of the $\mathrm{O}_{2}$ molecule physisorbed at the $H$ site of the $(6,6)$ SWNT as described by the inset. Spin-up and spin-down bands are shown by dash-dotted and continuous lines, respectively. The zero of energy is taken at $E_{F}$.

For example, since single oxygen (per unitcell) chemisorbed at the a site gives rise to flat bands below $E_{V}$, the band gap of $(8,0)$ SWNT remains unaffected. However, as a well known and usual effect of oxygen, the band gap of $(8,0)$ tube opens up to $3.64 \mathrm{eV}$ when all a-sites are filled by the chemisorbed oxygen atom.

\section{DISCUSSION AND CONCLUSIONS}

In this paper we studied the interaction between carbon nanotube and oxygen and resulting effects on the electronic structure. The physisorption of $\mathrm{O}_{2}$ molecules and chemisorption of $\mathrm{O}$ atoms are investigated for different coverage and adsorption patterns. Our results obtained from spin polarized calculations reveal the following: (i) $\mathrm{An}_{2}$ molecule can be adsorbed at different sites on a SWNT with comparable binding energies. Adsorption at the $T$ site of a $(8,0)$ tube is found to be energetically most favorable. The ground state of the adsorbed $\mathrm{O}_{2}$ molecule is the triplet state with a net magnetic moment of $2 \mu_{B}$ Bohr magneton. The binding energies calculated by including short and long range interactions are small and characteristics of physisorption. (ii) Stable chain formation and also a uniform coverage of $\mathrm{O}_{2}$ molecules in the triplet ground state on a s-SWNT are also possible. Since physisorbed $\mathrm{O}_{2}$ has net magnetic moment in the ground state, new magnetic molecules (or nanomagnets) can be constructed as a result of $\mathrm{O}_{2}$ decoration. (iii) GGA electronic structure calculations performed for optimized atomic geometry corresponding to the triplet ground state indicate that the unoccupied $O p p \pi^{*}(\downarrow)$ bands (states) of $\mathrm{O}_{2}$ occur $0.2-0.4$ $\mathrm{eV}$ above the top of the valence band of the s-SWNT. Only the row of $\mathrm{O}_{2}$ among different patterns studied in this paper yields a band gap as small as $25 \mathrm{meV}$. No matter how the coverage and pattern of adsorbed $\mathrm{O}_{2}$ are, the Fermi level always occurs above but close to $E_{V}$. The energy position of $O p p \pi^{*}(\downarrow)$ bands are robust and are not affected by small shift of the GGA optimized equilibrium position of $\mathrm{O}_{2}$ under 
van der Waals attraction. According to these results the hole doping of the s-SWNT by the physisorption of $\mathrm{O}_{2}$ is not valid. This conclusion is also corroborated by Derycke et al. ${ }^{7}$ who showed that the main effect of oxygen physisorption is not to dope the bulk of the tubes but to modify the barriers of the metal-semiconductor contact. It should be noted that the metallization of the tube can occur only by the lowering of $O p p \pi^{*}(\downarrow)$ states and dipping into the valence band of the $\mathrm{s}-\mathrm{SWNT}$. As a result, charge is transferred from the s-SWNT to the molecule, whereby the physisorption state changes into a chemisorption state. This argument is corroborated by the fact that the same $p p \pi^{*}(\downarrow)$ level of a single $\mathrm{O}_{2}{ }^{-}$molecule is lowered significantly. It appears that the lowering of $O p p \pi^{*}(\downarrow)$ state and hence electron transfer from C atoms to $\mathrm{O}_{2}$ does not occur by itself; it may be prevented by a kinetic barrier. ${ }^{46}$ Perhaps adsorption at a different environment, such as a defect site and impurity, or other factors help to overcome the kinetic barrier. (iv) The metallic $(6,0)$ armchair tube remains to be metallic after the physisorption of $\mathrm{O}_{2}$. (v) The characters of binding and range of the binding energy of $\mathrm{O}_{2}$ adsorbed on the metallic $(6,6)$ armchair tube are similar to those on the semiconducting $(8,0)$ tube. (vi) Dissociation of the $\mathrm{O}_{2}$ molecule and then chemisorption of individual $\mathrm{O}$ atoms is an exothermic process. However, an $\mathrm{O}_{2}$ molecule physisorbed on a perfect $(8,0)$ tube cannot dissociate by itself at low temperature, owing to a finite activation energy. Our calculations predict that individual $\mathrm{O}$ atoms are adsorbed preferably on top of the $\mathrm{C}-\mathrm{C}$ bonds and bind with $\mathrm{C}$ atoms by forming directional $\mathrm{O}-\mathrm{C}$ bonds. On the other hand, a metal atom like $\mathrm{Al}$ favors the $H$ site, leading to non-directional bonds between metal atom and the SWNT. ${ }^{17,49}$ The axial C-C bond survives after an oxygen atom is chemisorbed on top of this bond. In contrast, upon $\mathrm{O}$ chemisorption the zigzag $\mathrm{C}-\mathrm{C}$ bond is broken while $\mathrm{C}-\mathrm{O}$ bond is strengthened. The oxidation of the nanotube in the conventional sense starts with the breaking of the strained zigzag $\mathrm{C}-\mathrm{C}$ bonds upon the chemisorption of $\mathrm{O}$. The band gap of the $(8,0)$ tube is further opened at a high $\mathrm{O}$ coverage.

Note added in proof: We calculated the physisorption state of $\mathrm{O}_{2}$ at the $z$-site to be $\sim 0.85 \mathrm{eV}$ energetically more favorable than the chemisorption state in Ref. 50, which was brought to our attention recently. We note that this chemisorption state is neither easily accessible from the physisorption state, nor conforming to the hole doping picture because of its energy band gap of $\sim 0.5 \mathrm{eV}$.

\section{ACKNOWLEDGMENTS}

This work was partially supported by the National Science Foundation under Grant No. INT01-15021 and TÜBITAK under Grant No. TBAG-U/13(101T010). O.G. acknowledges the support by NATO Grant No. SfP971970, Turkish Department of Defense Grant No. KOBRA-001, and Thales JP8.04. S.C. acknowledges helpful discussions with Professor S. Süzer, and also partial financial support from Academy of Science of Turkey.

${ }^{1}$ S. Iijima, Nature (London) 354, 56 (1991); S. Iijima, T. Ichihashi, and Y. Ando, ibid. 356, 776 (1992).

${ }^{2}$ M. S. Dresselhaus, G. Dresselhaus, and P. C. Eklund, Science of Fullerenes and Carbon Nanotubes (Academic Press, San Diego, 1996).

${ }^{3}$ J. W. G. Wildör, L. C. Venema, A. G. Rinzler, R. E. Smalley, and C. Dekker, Nature (London) 391, 59 (1998); T. W. Odom, J. Huang, P. Kim, and C. M. Lieber, ibid. 391, 62 (1998).

${ }^{4}$ X. Blase, L. X. Benedict, E. L. Shirley, and S. G. Louie, Phys. Rev. Lett. 72, 1878 (1994).

${ }^{5}$ O. Gülseren, T. Yildirim, S. Ciraci, and C. Kilic, Phys. Rev. B 65, 155410 (2002); O. Gülseren, T. Yildirim, and S. Ciraci, ibid. 65, 153405 (2002).

${ }^{6}$ R. Martel, V. Derycke, C. Lavoie, J. Appenzeller, K. K. Chan, J. Tersoff, and Ph. Avouris, Phys. Rev. Lett. 87, 256805 (2001).

${ }^{7}$ V. Derycke, R. Martel, J. Appenzeller, and Ph. Avouris, Appl. Phys. Lett. 80, 2773 (2002).

${ }^{8}$ J. Park and P. L. McEuen, Appl. Phys. Lett. 79, 1363 (2001).

${ }^{9}$ A. Bezryadin, A. R. M. Verschueren, S. J. Tans, and C. Dekker, Phys. Rev. Lett. 80, 4036 (1998).

${ }^{10}$ L. Chico, V. H. Crespi, L. X. Benedict, S. G. Louie, and M. L. Cohen, Phys. Rev. Lett. 76, 971 (1996).

${ }^{11}$ M. Bockrath, D. H. Cobden, P. L. McEuen, N. G. Chopra, A. Zettl, A. Thess, and R. E. Smalley, Science 275, 1922 (1997).

${ }^{12}$ C. Kilic, S. Ciraci, O. Gülseren, and T. Yildirim, Phys. Rev. B 62, 16345 (2000).

${ }^{13}$ J. Kong, J. Cao, H. Dai, and E. Anderson, Appl. Phys. Lett. 80, 73 (2002).

${ }^{14}$ T. Yildirim, O. Gülseren, and S. Ciraci, Phys. Rev. B 64, 075404 (2001).

${ }^{15}$ J. Kong, N. R. Franklin, C. Zhou, M. G. Chapline, S. Peng, K. Cho, and H. Dai, Science 287, 622 (2000).

${ }^{16}$ P. G. Collins, K. Bradley, M. Ishigami, and A. Zettl, Science 287, 1802 (2000).

${ }^{17}$ O. Gülseren, T. Yildirim, and S. Ciraci, Phys. Rev. Lett. 87, 116802 (2001).

${ }^{18}$ O. Gülseren, T. Yildirim, and S. Ciraci, Phys. Rev. B 66, 121401 (2002).

${ }^{19}$ K. A. Dean and B. R. Chalamala, J. Appl. Phys. 85, 3832 (1999); Appl. Phys. Lett. 76, 375 (2000).

${ }^{20}$ P. M. Ajayan, T. W. Ebbesen, T. Ichihashi, S. Iijima, K. Tanigaki, and H. Hiura, Nature (London) 362, 522 (1993).

${ }^{21}$ T. W. Ebbesen, P. M. Ajayan, H. Hiura, and K. Tanigaki, Nature (London) 367, 519 (1994).

${ }^{22}$ K. Morishita and T. Takarada, Carbon 35, 977 (1997).

${ }^{23}$ S. H. Jhi, S. G. Louie, and M. L. Cohen, Phys. Rev. Lett. 85, 1710 (2000).

${ }^{24}$ S. M. Lee, Y. H. Lee, Y. G. Hwang, J. R. Hahn, and H. Kang, Phys. Rev. Lett. 82, 217 (1999).

${ }^{25}$ D. C. Sorescu, K. D. Jordan, and P. Avouris, J. Phys. Chem. B 105, 11227 (2001).

${ }^{26}$ X. Y. Zhu, S. M. Lee, Y. H. Lee, and T. Frauenheim, Phys. Rev. Lett. 85, 2757 (2000). 
${ }^{27}$ C.-Y. Moon, Y.-S. Kim, E.-C. Lee, Y.-G. Jin, and K. J. Chang, Phys. Rev. B 65, 155401 (2002).

${ }^{28}$ N. Park, S. Han, and J. Ihm, Phys. Rev. B 64, 125401 (1999).

${ }^{29}$ D. J. Mann and M. D. Halls, J. Chem. Phys. 116, 9014 (2002).

${ }^{30}$ A. Ricca and J. A. Drosco, Chem. Phys. Lett. 362, 217 (2002).

${ }^{31}$ P. Hohenberg and W. Kohn, Phys. Rev. 136, B864 (1964); W. Kohn and L. J. Sham, Phys. Rev. 140, A1133 (1965).

${ }^{32}$ M. C. Payne, M. P. Teter, D. C. Allen, T. A. Arias, and J. D. Joannopoulos, Rev. Mod. Phys. 64, 1045 (1992).

${ }^{33}$ J. P. Perdew and Y. Wang, Phys. Rev. B 46, 6671 (1992).

${ }^{34}$ D. Vanderbilt, Phys. Rev. B 41, 7892 (1990).

${ }^{35}$ H. J. Monkhorst and J. D. Pack, Phys. Rev. B 13, 5188 (1976).

${ }^{36}$ J. P. Perdew, J. A. Chevary, S. H. Vosko, K. A. Jackson, M. R. Jackson, M. R. Pederson, D. J. Singh, and C. Fiolhais, Phys. Rev. B 46, 6671 (1992).

${ }^{37}$ G. Kern, G. Kresse, and J. Hafner, Phys. Rev. B 59, 8551 (1999).

${ }^{38}$ Y. Baskin and L. Meyer, Phys. Rev. 100, 544 (1955).

${ }^{39}$ A. Janotti, S. H. Wei, and D. J. Singh, Phys. Rev. B 64, 174107 (2001).

${ }^{40}$ J. Furthmüller, J. Hafner, and G. Kresse, Phys. Rev. B 50, 15606 (1994).
${ }^{41}$ E. M. Lifshitz, Zh. Éksp. Teor. Fiz. 29, 94 (1956) [Sov. Phys. JETP 2, 73 (1956)].

${ }^{42} \mathrm{~J}$. N. Israelachvili, Intermolecular and Surface Forces (Academic, London 1985).

${ }^{43}$ S. Ciraci, E. Tekman, A. Baratoff, and I. P. Batra, Phys. Rev. B 46, 10411 (1992).

${ }^{44}$ T. A. Halgren, J. Am. Chem. Soc. 114, 7827 (1992).

${ }^{45}$ K. P. Huber and G. Herzberg, Molecular Spectra and Molecular Structure. IV. Constants of Diatomic Molecules (Van Nostrand Reinhold, New York, 1979).

${ }^{46}$ H. Ulbricht, G. Moos, and T. Hertel, Phys. Rev. B 66, 075404 (2002).

${ }^{47}$ Y. Wu, N. Marzari, and R. Car, Bull. Am. Phys. Soc. 47, 1088 (2002).

${ }^{48}$ I. W. Chiang, B. E. Brinson, R. E. Smalley, J. L. Magrave, and R. H. Hauge, J. Phys. Chem. B 105, 1157 (2001).

${ }^{49}$ V. M. K. Bagci, O. Gülseren, T. Yildirim, Z. Gedik, and S. Ciraci, Phys. Rev. B 66, 045409 (2002).

${ }^{50}$ S. P. Chan, G. Chen, X. G. Gong, and Z. F. Liu, Phys. Rev. Lett. 90, 086403 (2003). 